\title{
Abordagem Atividade-Intensidade para o Reconhecimento de Atividades
}

\author{
Eduardo Gomes ${ }^{1}$, Natália Melgaço Pereira Torres ${ }^{1}$, Izabela Mocaiber ${ }^{2}$, Danilo \\ Artigas $^{1}$, Alessandro Copetti ${ }^{1}$ \\ ${ }^{1}$ Instituto de Ciência e Tecnologia - Universidade Federal Fluminense (UFF) \\ 28.895-532 - Rio das Ostras - RJ - Brasil \\ ${ }^{2}$ Instituto de Humanidades e Saúde - Universidade Federal Fluminense (UFF) \\ 28.895-532 - Rio das Ostras - RJ - Brasil \\ eduardosg2@gmail.com, natalia.melgaco@gmail.com, mocaiber@vm.uff.br, \\ daniloartigas@puro.uff.br, alessandro.copetti@gmail.com
}

\begin{abstract}
In pervasive healthcare monitoring, a critical application in support to the patient is activity recognition. Despite the great number of studies on this topic, a parameter that has received less attention is intensity recognition. In the present work, we propose the coupling between activity and intensity, namely, Activity-Intensity, in accelerometer data to better describe daily activities of patients. Additionally, we investigated the feasibility of Fuzzy Logic in activity recognition. Results showed the viability of the classification and the good accuracy of Activity-Intensity recognition.
\end{abstract}

Resumo. No monitoramento em saúde pervasivo, uma aplicação essencial no acompanhamento do dia a dia da pessoa é o reconhecimento de atividades. Apesar dos diversos estudos sobre esse tema, um parâmetro pouco considerado é o reconhecimento da intensidade. Neste trabalho, propomos o acoplamento da atividade com a intensidade, a qual denominamos Atividade-Intensidade, em dados obtidos de acelerômetros, para melhor descrever as atividades diárias de um paciente. Adicionalmente, investigamos iniciativas de Lógica Fuzzy no reconhecimento de atividades. Os resultados mostraram a viabilidade da classificação e o bom desempenho do reconhecimento Atividade-Intensidade.

\section{Introdução}

Com o avanço das tecnologias de sensores sem fio, posicionados tanto nos ambientes como no corpo das pessoas (computação vestível), está sendo possível projetar novos mecanismos de monitoramento remoto de pacientes e aperfeiçoar tanto a análise quanto a acurácia dos sistemas. No monitoramento em saúde, uma aplicação essencial no acompanhamento do dia a dia da pessoa é o reconhecimento de atividades como dormir, sentar, caminhar, varrer e etc. Uma forma de efetuar esse reconhecimento é com o auxílio dos dados fornecidos por sensores do tipo acelerômetro [Bulling et al. 2014] [Li et al. 2009] [Helmi et al., 2009].

Um aspecto que não tem sido abordado em trabalhos que envolvem reconhecimento de atividades é o reconhecimento do nível de intensidade da atividade em questão. Geralmente a interpretação dada para a intensidade é em relação ao esforço que a atividade proporciona. Neste trabalho, enfatizamos a necessidade de se considerar os 
movimentos que são realizados durante uma atividade. Em trabalhos da área de Saúde, a intensidade é estratificada em termos de atividade leve, moderada e vigorosa [Ainsworth et al. 2000]. Nesse sentido, é importante acoplar esse parâmetro ao reconhecimento de atividades. $\mathrm{Na}$ área biomédica, a intensidade de movimentos tem sido determinada com métodos fisiológicos específicos que envolvem o cálculo do gasto energético. Entretanto, em determinadas abordagens, é importante ter a informação da intensidade do movimento através de métodos mais simples e sem o interesse principal, com o gasto energético.

Com esse acoplamento da atividade com a intensidade, a qual denominamos Atividade-Intensidade, é possível representar melhor o conhecimento de profissionais de saúde, na forma de sistemas de decisão com regras de especialistas. Exemplos de regras em um sistema de decisão, como apresentado em [Copetti et al. 2013], podem ser estabelecidos: a) se a atividade é doméstica, a intensidade é vigorosa e a frequência cardíaca é alta, então a situação do Paciente é de alerta (e não de emergência). Com isso, as alterações nos sinais vitais influenciadas por mudanças no comportamento poderiam ser toleradas com base em regras que levem esses fatores em consideração. Em outro exemplo, se a atividade é dormindo e a intensidade é vigorosa, então incrementa a possibilidade de insônia. Os dois exemplos mostram então uma intensidade explícita enquanto a pessoa realiza a atividade.

O presente trabalho investiga o impacto da discriminação da intensidade no reconhecimento de atividades distintas. Assim, propomos o acoplamento AtividadeIntensidade para aperfeiçoar os mecanismos de reconhecimento de atividade. Adicionalmente, investigamos iniciativas de Lógica Fuzzy no reconhecimento de atividades. A justificativa por essa técnica se dá por permitir expressar o conhecimento de especialistas no seu conjunto de regras e eventualmente beneficiar a inferência da Atividade-Intensidade.

O trabalho está estruturado como segue: a seção 2 apresenta trabalhos na área de Reconhecimento de Atividades, dando ênfase para trabalhos que já fizeram uso de Lógica Fuzzy como mecanismo de inferência ou de classificação. A seção 3 aborda o Reconhecimento de Atividades acoplado a intensidade dos movimentos. A seção 4 apresenta os testes com essa abordagem. Por fim, são apresentadas as conclusões.

\section{Trabalhos Relacionados em Reconhecimento da atividade}

Os sistemas de monitoramento para reconhecimento da atividade são ferramentas essenciais para os profissionais de saúde. Os sistemas podem propiciar intervenções precoces, e atuar tanto na reabilitação quanto na prevenção. Podemos imaginar sistemas de reconhecimento de atividades que analisem informações sobre quantos e em quais momentos o paciente experimenta uma alteração em sua rotina física. Uma aplicação prática pode ser a identificação e a análise de momentos de falta de mobilidade. Em uma situação contrária, pode ser identificado, por exemplo, o excesso de tremores [Palmerini et al. 2011].

Muitos trabalhos [bin Abdullah et al. 2012] [Gjoreski, H. et al. 2015] [Li 2009] já usam técnicas para reconhecimento de atividades das pessoas, principalmente, com o uso de algoritmos de aprendizado de máquina. Recentemente, algumas pesquisas têm direcionado para contextos específicos de saúde, como, por exemplo, envolvendo o autismo [Albinali 2009], reabilitação [Pärkkä 2009] e hipertensão [Copetti 2009]. Essa diversidade de áreas de aplicação na saúde faz com que a interação tenha que ser maior entre o conhecimento do especialista e o projetista do sistema. Portanto, há a necessidade de 
modelos que consigam expressar conceitos e regras, com fácil interpretação por parte do especialista.

No trabalho de [Copetti et al. 2013] a Lógica Fuzzy foi usada para modelar variáveis relacionadas a dados fisiológicos associados, em alto nível, com o comportamento. Nesse trabalho, alterações nos sinais vitais (elevação da frequência cardíaca) influenciados por mudanças no comportamento (ex: realização de uma atividade como varrer) puderam ser tolerados como dentro da normalidade.

No trabalho de [Helmi 2009] foi utilizado Lógica Fuzzy para reconhecer quatro atividades distintas utilizando um acelerômetro triaxial na cintura do voluntário. As atividades estudadas foram: ir para frente, saltar, subir escada e descer escada. Nesse estudo, foi observado que a Lógica Fuzzy obteve um desempenho melhor do que os outros classificadores, tendo um total de $95 \%$ de acurácia no reconhecimento das atividades.

No trabalho de [Chiang 2012] o sistema de Lógica Fuzzy foi utilizado no reconhecimento de atividades corporais para auxiliar na fisioterapia de pacientes com AVC (acidente vascular cerebral) e na fisioterapia geriátrica. Para coletar os movimentos do corpo humano utilizou-se uma placa com acelerômetro e giroscópio no tórax e outra na coxa e as atividades avaliadas foram deitar, sentar, levantar, andar e correr. A acurácia total no reconhecimento das atividades alcançou mais de $96 \%$.

No trabalho de [Yang 2008] foi utilizado uma rede Fuzzy-Bayesiana para reconhecer as atividades dos voluntários no seu dia-a-dia através de um acelerômetro 2D no antebraço e mais quatro sensores fisiológicos. Foram nove atividades do cotidiano analisadas: caminhar, correr, se exercitar, comer, ler, estudar, brincar, descansar e dormir. A acurácia obtida foi de $74,4 \%$ no reconhecimento das atividades, incluindo atividades dinâmicas e atividades estáveis.

Já o trabalho de [Liu e Chang 2009] realiza o reconhecimento das atividades andando, sentando e caindo. A técnica neuro-fuzzy foi utilizada e 3 acelerômetros foram posicionados no corpo no peito, na cintura e na coxa. A acurácia obtida foi de $88,7 \%$.

Nos trabalhos discutidos de [Helmi 2009] [Chiang 2012] [Yang 2008], existe uma complexidade em definir conjuntos para as variáveis Fuzzy de entrada e de saída, pois o problema do reconhecimento da atividade depende do conhecimento dos dados (em contraposição ao conhecimento dos especialistas). Assim, é necessário criar conjuntos Fuzzy bem definidos para as variáveis de entrada, as quais são as acelerações dos eixos ou são medidas estatísticas aplicadas a essas acelerações. Já a variável de saída atividade, apresentada em [Helmi 2009] e [Chiang 2012] como uma sequência de conjuntos fuzzy cada conjunto é uma atividade - é uma forma que viola o benefício da sobreposição de conjuntos Fuzzy. Já a técnica de Neuro-Fuzzy, utilizada no trabalho de [Liu e Chang 2009], considera o conhecimento dos dados para ajustar os pesos sinápticos da rede neural na fase de treinamento, o que é adequado para o problema. No entanto, a técnica perde a interpretabilidade das regras por extrapolar as combinações em razão das entradas.

O nosso trabalho apresenta uma nova abordagem, complementar a forma como é tratado o reconhecimento de atividades dos trabalhos discutidos nessa seção, integrando a intensidade dos movimentos à atividade sendo executada, isto é, abordagem AtividadeIntensidade. Para nosso conhecimento, essa abordagem não foi explorada até o momento, como pode ser verificado nos estudos de área, como, [bin Abdullah et al. 2012] [Bulling et al. 2014] [Gjoreski, H. et al. 2015], bem como trabalhos específicos contemplando uma 
interpretação da intensidade diferente da adotada aqui [Ribeiro Filho et al. 2016] [Reiss 2014], discutidos na seção 3 .

\section{Reconhecimento de Atividades Acoplado à Intensidade dos Movimentos}

A intensidade de uma atividade, sob a perspectiva do esforço fisiológico dispendido, é habitualmente determinada pelo Gasto Energético Metabólico ou MET. O MET (Metabolic Energy Expenditure) [Ainsworth et al. 2000] reflete o consumo energético associado à execução de uma atividade. O MET representa por definição a proporção entre a taxa metabólica durante o trabalho físico e a taxa metabólica basal padrão de 1,0.1 MET é considerado a taxa metabólica em repouso enquanto sentado quieto. Então, de maneira organizada e sistematizada, pode-se estabelecer diferentes níveis de intensidades (leve: <3,0 METs; moderado: 3 - 6 METs e vigoroso (>6 METs). São atribuídos níveis de intensidade à cada atividade em função do gasto energético expresso em METs. Essa classificação de esforço depende do tipo de atividade e do grau de intensidade da mesma, gerando um valor representativo (ex. andar de bicicleta: 8,0 METs e meditar : 1,0 MET). Assim, a intensidade do movimento recebe geralmente uma interpretação relativa ao gasto energético [Ainsworth et al. 2000]. Observa-se aqui que a variável intensidade pode ser naturalmente modelada como variável Fuzzy, e ainda fazer parte de regras em sistemas de decisão em saúde.

A atividade física é definida como qualquer movimento corporal realizado pela musculatura esquelética que leve a algum gasto acima do repouso [Caspersen et al., 1985]. Assim, a quantidade de energia gasta na execução do movimento é o critério usado para diferenciar a atividade física. A medição do gasto energético é amplamente discutida no meio científico por meio de consumo de oxigênio, frequência cardíaca e acelerômetros [Melanson e Freedson, 1996]. Apesar da complexidade da medida da atividade física, instrumentos acessíveis e de baixo custo são de interesse de pesquisadores e clínicos. Nesse sentido, a utilização de sensores de movimento parecem ser interessantes, e se apoiam na ideia de que movimentos corporais sejam reflexo do gasto energético [Melanson e Freedson, 1996].

$\mathrm{Na}$ área de Computação Pervasiva, alguns trabalhos seguem essa interpretação de esforço para a intensidade. [Ribeiro Filho et al. 2015] e [Ribeiro Filho et al. 2016] propõem um sistema de reconhecimento de atividades e medição de sua intensidade. Um experimento foi desenvolvido com 3 acelerômetros posicionados na coxa, no peito e na cintura. As atividades analisadas incluem correndo, subindo e descendo escadas, andando, em pé, sentado e deitado. A taxa de acerto ficou em torno de $82 \%$. A frequência cardíaca é usada para medir a intensidade, no entanto, o tratamento e a análise desses dados não são discutidos para uma classificação da intensidade.

Já no trabalho de [Reiss 2014], variações de Adaptive Boosting são usadas como técnicas para estimar a intensidade da atividade nas três categorias (leve, moderado e vigoroso). Usando a base de dados PAMAP2 [Reiss \& Stricker 2012], a qual possui dados de atividades monitoradas com três acelerômetros e monitor de frequência cardíaca, são obtidos resultados próximos a $90 \%$ para estimar a intensidade. Por exemplo, atividades como estar sentado, trabalhar no computador e assistir TV são classificadas como de esforço leve.

No entanto, neste trabalho estamos interessados na movimentação (oscilação) da 
pessoa durante uma atividade, isto é, na interação atividade $\mathrm{x}$ intensidade. Adotamos a mesma classificação de intensidade, mas sem associar ao gasto energético. Alguns exemplos motivadores de situações para adoção da abordagem Atividade-Intensidade são:

- movimentação excessiva durante o sono: a pessoa mesmo estando deitada, apresenta níveis diferentes de movimentação que deveriam ser identificados para saber, por exemplo, qual a intensidade dos movimentos no último minuto.

- ritmo da caminhada que difere do habitual: a atividade de andar pode apresentar características a serem exploradas por especialistas quanto a análise da caminhada.

- permanência prolongada em um sofá: estar sentado pode revelar momentos que a pessoa adormece ou que realiza tarefas manuais.

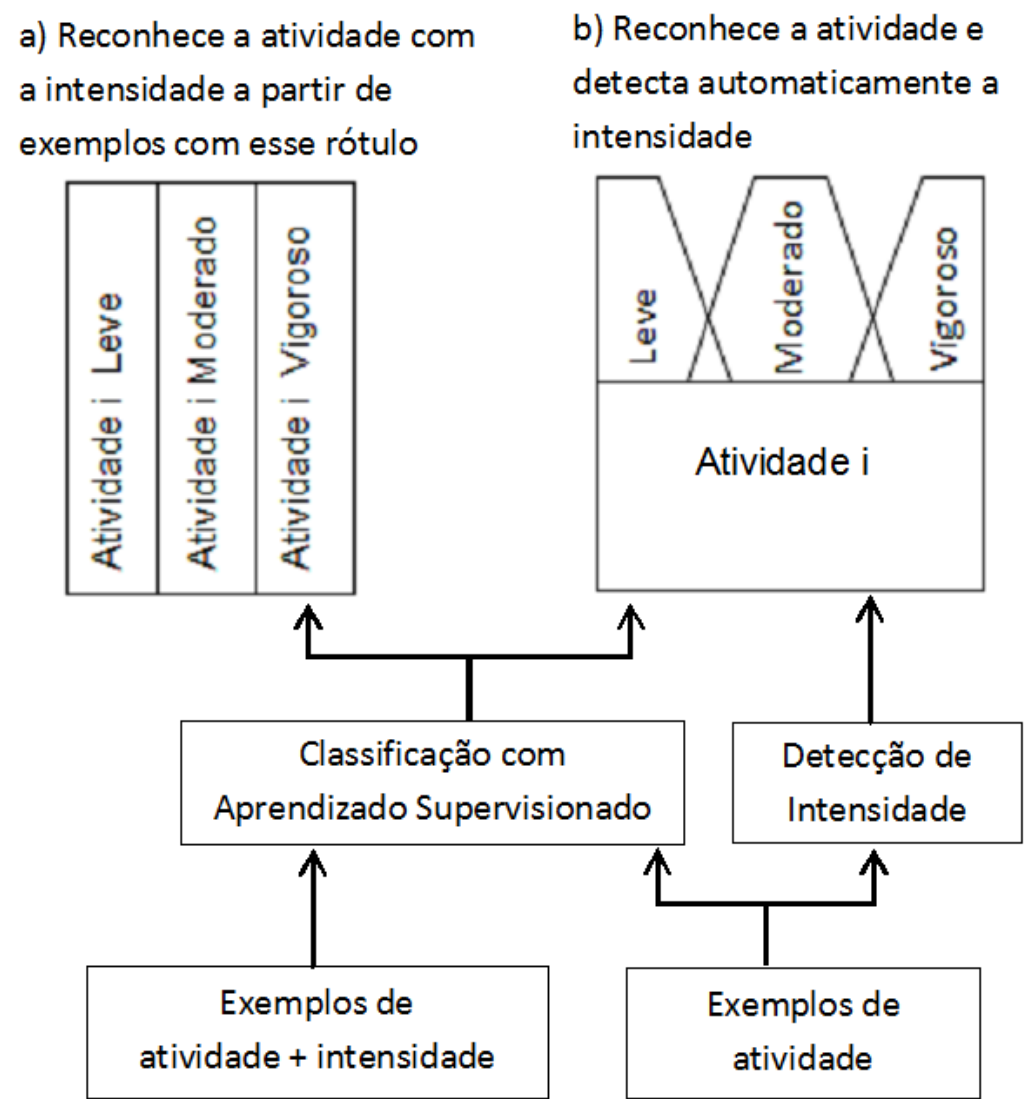

Figura 4.1 Proposta de abordagem Atividade-Intensidade

A Figura 4.1 apresenta a abordagem proposta de Atividade-Intensidade com duas opções para obter esse acomplamento. Na opção 'a', os exemplos usados no treinamento são rotulados com a atividade+intensidade. Essa forma é adotada nos experimentos da seção 4. Na opção 'b', o exemplo possui o rótulo somente da atividade. Em seguida, há a necessidade de uma técnica para detectar os limites entre intensidades, por exemplo, agrupamento em 3 clusters ou análise estatística dos dados. Os limites também podem ser usados para definir funções de pertinência para uma variável fuzzy. 


\section{Experimentos e Análise dos Resultados}

O principal objetivo do experimento foi investigar se é possível obter bons resultados no reconhecimento da intensidade de determinadas atividades. Neste trabalho, investigamos 3 atividades: deitado, sentado e andando. As atividades foram escolhidas por serem as mais corriqueiras de um idoso em seu domicílio. Cada atividade tem 3 tipos de intensidade avaliados: leve, moderado e vigoroso. Dessa forma, totalizando 9 possíveis situações. De maneira detalhada, cada situação corresponde as seguintes exemplos do dia a dia: Deitado leve: todo corpo parado na cama; Deitado moderado: em certo tempo vira o corpo para mudar de posição; Deitado vigoroso: simulação de uma insônia crônica onde todos os membros inclusive a cabeça alteram a posição inicial; Sentado leve: membros inferiores totalmente relaxados no assento; Sentado moderado: pernas inquietas e braços praticamente relaxados; Sentado vigoroso: membros inferiores e superiores ativos; Andar leve: caminhada lenta; Andar moderado: caminhada normal; Andar vigoroso: caminhada com passos rápidos.

O experimento consistiu na coleta de dados de 5 voluntários (4 homens e 1 mulher) na faixa de 15 a 50 anos. Cada voluntário possuía um celular posicionado dentro de uma pochete fixa na cintura conforme Figura 4.2. Os voluntários foram orientados a reproduzir cada uma das situações. Durante essas reproduções, o acelerômetro 3D presente no celular registrou informações da aceleração da gravidade do dispositivo e do tempo. O celular utilizado possui processador de $1 \mathrm{GHz}, 1 \mathrm{~GB}$ de memória RAM, com SO Android 4.1.2.

O tempo de coleta de dados no celular foi de 1 minuto para cada uma das 9 situações. A segmentação foi aplicada com uma janela de $2,5 \mathrm{~s}$ (sem sobreposição). Na extração de características, as medidas estatísticas aplicadas incluíram: média, mediana, moda, variância, desvio padrão e RMS. Em seguida, os dados foram discretizados, possibilitando um tratamento por faixas numéricas. Devido aos motivos apresentados na seção 2 sobre Lógica Fuzzy aplicada no reconhecimento da atividade, a técnica de classificação utilizada foi a de Redes Neurais. A validação utilizou validação cruzada de 10 partições.

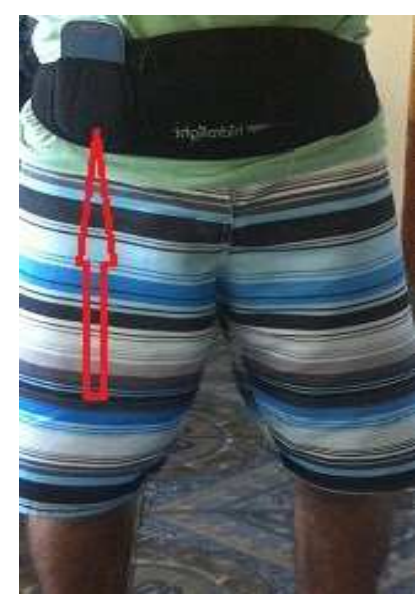

Figura 4.2 Posição do celular no corpo do voluntário.

O primeiro teste verifica o desempenho do reconhecimento para o acoplamento Atividade-Intensidade. A Figura 4.3 apresenta a acurácia para todas as 9 situações. Os melhores resultados são para as atividades de deitado e sentado. Nessas atividades, se analisarmos as intensidades (leve, moderado e vigoroso) vemos que não apresentam 
variações significativas, além de não ser possível identificar a intensidade que obteve os piores resultados. Esses resultados mostram a viabilidade de integrar atividade com intensidade do movimento.

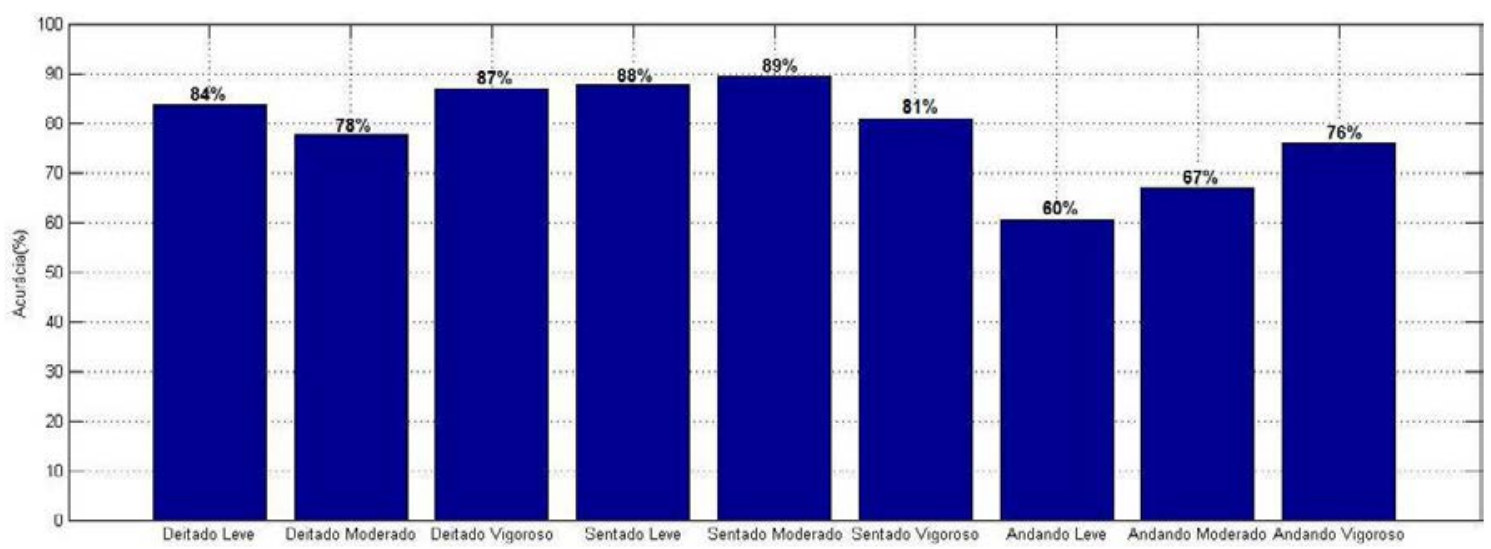

Figura 4.3 Acurácia nas 9 situações de Atividade-Intensidade.

Para avaliar mais detalhadamente a atividade de andando, a Figura 4.4 mostra a matriz de confusão obtida. Com a matriz é possível ver que a atividade de 'andando' não obteve resultados ótimos em comparação com as demais atividades. A diferença no ritmo da caminhada de cada voluntário (mais lento ou mais rápido) pode explicar esse resultado. Um fato que comprova essa observação na matriz de confusão é o erro sempre quantificado entre vizinhos de intensidade e nunca entre o 'leve' e o 'vigoroso', e um número reduzido de 9 casos que foram classificados erroneamente como 'SentadoVigoroso', mas deveriam ser 'AndandoLeve'.

\begin{tabular}{|c|c|c|c|c|c|c|c|c|c|c|}
\hline a & $\mathrm{b}$ & c & d & e & f & $g$ & $\mathrm{~h}$ & $i$ & $<--$ & classified as \\
\hline 105 & 15 & 2 & 0 & 1 & 0 & 0 & 0 & 0 & a & $=$ DeitadoLeve \\
\hline 11 & 94 & 16 & 0 & 0 & 0 & 0 & 0 & 0 & $\mathrm{~b}$ & $=$ DeitadoModerado \\
\hline 3 & 15 & 104 & 0 & 0 & 0 & 0 & 0 & 0 & c & = DeitadoVigoroso \\
\hline 0 & 0 & 0 & 105 & 11 & 4 & 2 & 0 & 0 & d & $=$ SentadoLeve \\
\hline 0 & 1 & 0 & 7 & 105 & 7 & 1 & 0 & 0 & । & $=$ SentadoModerado \\
\hline 0 & 0 & 0 & 2 & 5 & 98 & 13 & 3 & 0 & f & = SentadoVigoroso \\
\hline 0 & 0 & 0 & 1 & 0 & 9 & 80 & 26 & 4 & g & = AndandoLeve \\
\hline 0 & 0 & 0 & 1 & 0 & 2 & 19 & 83 & 16 & $\mathrm{~h}$ & $=$ AndandoModerado \\
\hline 0 & 0 & 1 & 0 & 0 & 0 & 8 & 21 & 90 & i & $=$ AndandoVigoroso \\
\hline
\end{tabular}

Figura 4.4 Matriz de confusão da classificação das 9 situações.

Uma outra razão para os resultados menores na atividade de andando é devido ao posicionamento do acelerômetro. Testes realizados com um acelerômetro no pulso (embarcada em um relógio) resultaram em uma acurácia acima de $85 \%$.

A Figura 4.5 mostra a acurácia quando somente é considerada a atividade sem a intensidade. Esse cenário é considerado tradicional, apresentado na maior parte dos trabalhos relacionados. Vemos que a técnica obteve excelentes resultados. 


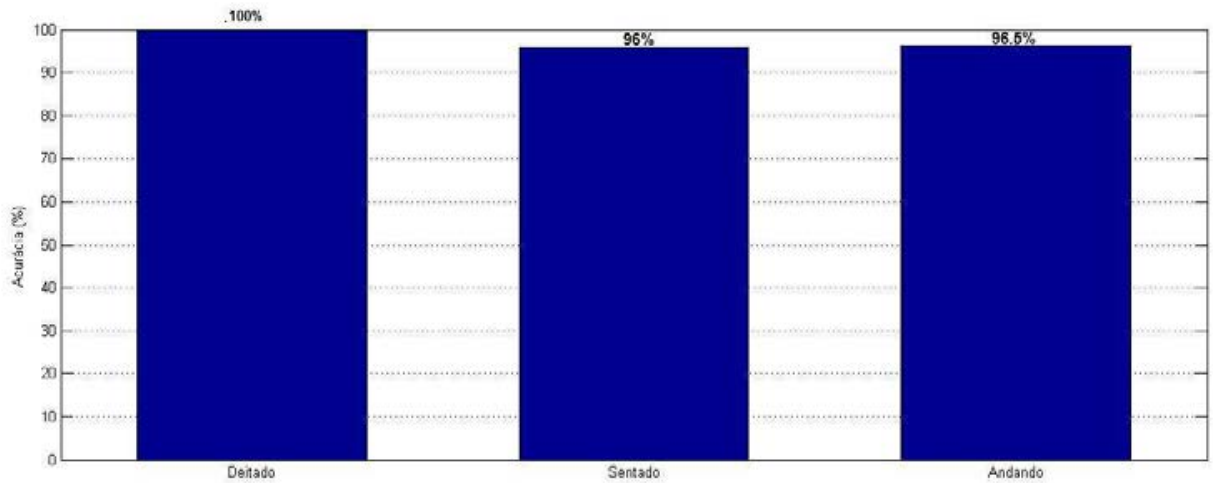

Figura 4.5 Acurácia considerando somente a atividade dos voluntários.

Para verificar os resultados com somente as situações que apresentam na prática um maior distanciamento em relação a intensidade dos movimentos, fizemos um teste somente com as situações: deitado leve, sentado moderado e andando vigoroso (Figura 4.6). Ou seja, são os exemplos mais representativos de situações mais equidistantes. $O$ teste mostra que a integração atividade-intensidade não deteriora o resultado, ao considerar situações extremas entre as 9 trabalhadas.

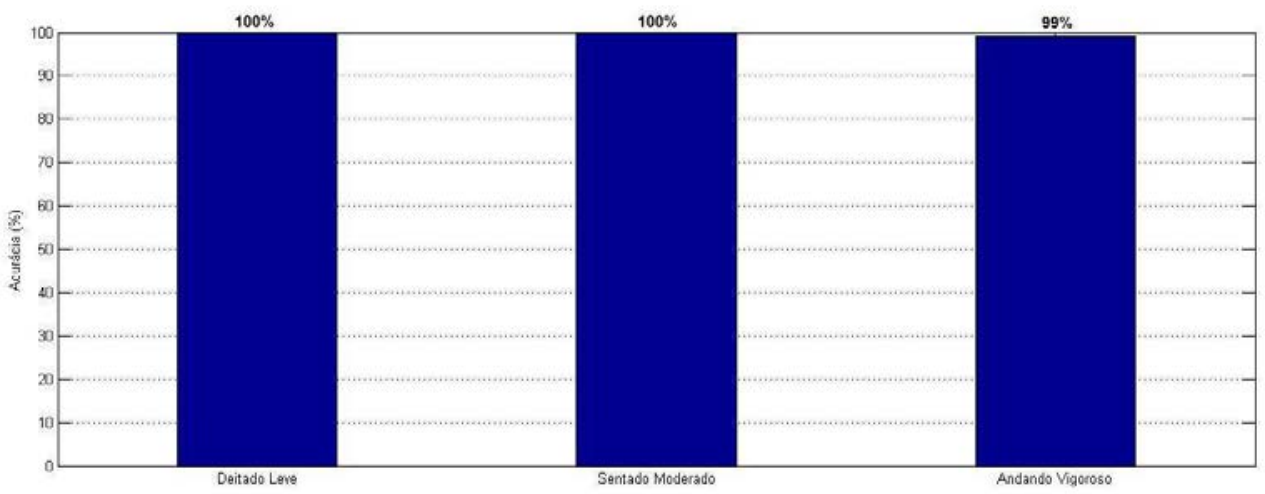

Figura 4.6 Classificação com dados de somente 3 situações que apresentam maior distanciamento em relação a intensidade dos movimentos.

Concluindo, vemos que, das três atividades que dominam o maior tempo da vida diária de idosos, a atividade de andar acoplada a intensidade é a que obteve a acurácia mais baixa (60\%-76\%, Figura 4.3). Esse resultado pode ser melhorado com o uso de mais acelerômetros ou o posicionamento do acelerômetro em outras partes do corpo. Ademais, mantendo nossa proposta de uso do smartphone, podemos treinar com dados somente do paciente (específico do usuário). Segundo [Bulling et al. 2014], o desempenho é geralmente mais alto do que o caso de ser independente do usuário.

\section{Conclusões}

O trabalho investigou a inserção do conceito da intensidade no reconhecimento de atividades, propondo a abordagem Atividade-Intensidade. A classificação utilizando essa abordagem obteve bons resultados: a acurácia média foi de $83 \%$ para atividade deitado; 
$86 \%$ para sentado; e $67,67 \%$ para andando. Nesse sentido, acreditamos que a abordagem Atividade-Intensidade forneça recursos para melhor descrever as atividades diárias de um paciente. A Lógica Fuzzy pode modelar de uma forma natural a variável intensidade, além de ser uma ferramenta interessante para sistemas de decisão envolvendo AtividadeIntensidade. Além disso, os resultados apresentados são de grande interesse pois esse mecanismo de classificação, baseado em acelerômetros, tem um custo muito baixo sendo portanto de fácil acesso.

Como trabalho futuros, pretendemos explorar ainda mais os resultados obtidos com o posicionamento de mais acelerômetros em diferentes partes do corpo, o que beneficia determinados tipos de atividades. Pretendemos, ainda, aplicar técnicas como a clusterização para agrupar os diferentes níveis de intensidade levando em conta a personalização, isto é, detectar os níveis adequados de intensidade para uma pessoa específica. De forma complementar, um Sistema Especialista Fuzzy será desenvolvido para analisar o comportamento de pacientes, agregando o classificador desenvolvido no presente trabalho.

\section{Agradecimentos}

Os autores gostariam de agradecer o apoio financeiro recebido da FAPERJ e da PROPPI/PDI/UFF.

\section{Referências Bibliográficas}

Ainsworth, B. E. et al. (2000). "Compendium of physical activities: an update of activity codes and MET intensities". Medicine and science in sports and exercise, 32(9; SUPP/1), S498-S504.

Albinali, F., Goodwin, M. S. and Intille, S. S. (2009) "Recognizing stereotypical motor movements in the laboratory and classroom: a case study with children on the autism spectrum”, Ubiquitous Computing, pp. 71-80.

Bulling, A., Blanke, U., \& Schiele, B. (2014). "A tutorial on human activity recognition using body-worn inertial sensors". ACM Computing Surveys (CSUR), 46(3), 33.

bin Abdullah, M. F. A., Negara, A. F. P., Sayeed, M. S., Choi, D. J., \& Muthu, K. S. (2012). Classification algorithms in human activity recognition using smartphones. International Journal of Computer and Information Engineering, 6, 77-84.

Caspersen, C. J., Powell, K. E., \& Christenson, G. M. (1985). Physical activity, exercise, and physical fitness: definitions and distinctions for health-related research. Public health reports, 100(2), 126.

Chiang, S. Y., Kan, Y. C., Tu, Y. C. and Lin, H. C. (2012) "Activity Recognition by Fuzzy Logic System in Wireless Sensor Network for Physical Therapy", Intelligent Decision Technologies, vol. 2, no. 16, pp. 191-200.

Copetti, A., Leite, J. C. B., Loques, O., da Nóbrega, A. C. L. and Barbosa, T. P. (2009) "Chequer. Intelligent Context-Aware Monitoring of Hypertensive Patients." In: Situation Recognition and Medical Data Analysis in Pervasive Health Environments. 
Pervasive Healthcare Conference, Londres, Reino Unido.

Copetti, A., Leite, J. C. B. and Loques, O. (2013) "A Decision-making Mechanism for Context Inference in Pervasive Healthcare Environments", Decision Support Systems, 55(2): 528-537.

Gjoreski, H. et al. (2015). Competitive Live Evaluations of Activity-Recognition Systems. Pervasive Computing, IEEE, 14(1), 70-77.

Helmi, M. and AlModarresi, S.M.T. (2009) "Human Activity Recognition Using a Fuzzy Inference System”. FUZZ-IEEE. Korea.

Li, Q., Stankovic, J. A., Hanson, M. A., Barth, A. T., Lach, J. and Zhou, G. (2009) "Accurate, fast fall detection using gyroscopes and accelerometer-derived posture information", Wearable and Implantable Body Sensor Networks, pp. 138-143.

Liu, S.H. and Chang, Y.J. (2009) "Using accelerometers for physical actions recognition by a neural fuzzy network", Telemedicine and e-Health, 15(9): 867-876.

Melanson Jr, E. L., Freedson, P. S., \& Blair, S. (1996). "Physical activity assessment: a review of methods". Critical Reviews in Food Science \& Nutrition, 36(5), 385-396.

Palmerini, L., Rocchi, L., Mellone, S., Valzania, F., \& Chiari, L. (2011). "Feature selection for accelerometer-based posture analysis in Parkinson's disease". Information Technology in Biomedicine, IEEE Transactions on, 15(3), 481-490.

Pärkkä, J. et al. (2009) "Relationship of psychological and physiological variables in longterm self-monitored data during work ability rehabilitation program", Information Technology in Biomedicine, 13(2): 141-151.

Reiss, A., \& Stricker, D. (2012). "Introducing a new benchmarked dataset for activity monitoring". In Symposium on Wearable Computers (ISWC), (pp. 108-109). IEEE.

Reiss, A. (2014). "Personalized mobile physical activity monitoring for everyday life" (Tese de Doutorado, Technical University of Kaiserslautern).

Ribeiro Filho, J. D. P., Silva, F. J. S., Coutinho, L. R. and Gomes, B. T. P. (2015) "MHARS: Sistema Móvel de Reconhecimento de Atividades em Ambient Assisted Living”, In: Simpósio Brasileiro de Computação Ubíqua e Pervasiva- SBCUP, Recife.

Ribeiro Filho, J. D. P., Silva, F. J. S., Coutinho, L. R., Gomes, B. T. P. and Endler, M. (2016) “A Movement Activity Recognition Pervasive System for Patient Monitoring in Ambient Assisted Living”, 31st ACM Symposium On Applied Computing (SAC), Health Track.

Yang, S. I. and Cho, S. B. (2008) "Recognizing human activities from accelerometer and physiological sensors" IEEE International Conference on Multisensor Fusion and Integration for Intelligent Systems. Seoul, Korea. 